\title{
STUDY OF FOOD ADDITIVES COMPOSITION IN COMMERCIALLY PROCESSED BEEF PRODUCTS
}

\author{
[Kajian Komposisi Bahan Tambahan Pangan dalam Produk-Produk \\ Daging Sapi Olahan Komersial]
}

\author{
Mirza Rizqi Zulkarnain ${ }^{1) *}$, Glorya Pricillia ${ }^{2)}$, and Yasmina Okinurshabani ${ }^{2)}$ \\ 1) Department of Food Technology, Faculty of Life Science, International University Liaison, Banten \\ 2) Program of Food Technology, Faculty of Life Science, International University Liaison, Banten
}

Received July $31^{\text {st }} 2020$ / Accepted June $21^{\text {st }} 2021$

\begin{abstract}
Beef is one of the favorite red meats containing various essential nutrients. In the processed beef products, food additives are used for multiple purposes including preservation, moisture and texture retention, improvement of flavor and stability of color. The purpose of this study is to identify the food additives composition of halal and local-based commercially processed beef products based on the label of the products and the Indonesian regulations. There were 40 various commercially processed beef products assessed in this study. The food additives that were observed were flavor enhancers, preservatives, stabilizers, antioxidants, acidity regulators, and colorants. The types of food additives used in beef sausage products were stabilizers $(30.00 \%)$, preservatives $(25.56 \%)$, flavor enhancers $(15.56 \%)$, colorants $(14.44 \%)$, antioxidants $(13.33 \%)$ and acidity regulator $(1.11 \%)$. In beef balls products there were stabilizers $(50.00 \%)$, flavor enhancers $(20.00 \%)$, preservatives $(15.00 \%)$, antioxidants, acidity regulators and colorants $(5.00 \%$ each). The additives in beef burgers products include stabilizers $(29.73 \%)$, preservatives $(24.32 \%)$, colorants $(21.62 \%)$, flavor enhancers $(13.51 \%)$, antioxidants and acidity regulators $(5.41 \%$ each). Corned beef products contain preservatives (32.00\%), flavor enhancers and antioxidants $(24.00 \%$ each), and stabilizers (20.00\%). Additionally, smoked beef products use preservatives $(50.00 \%)$, stabilizers $(37.50 \%)$ and antioxidants $(12.50 \%)$. The only food additives used in beef floss products was flavor enhancer $(100 \%)$. The study suggests that Government Regulations on food additives should be reconsidered. Although the additives are still allowed in Indonesian regulations at the present, food additives of which are included in the list of carcinogenic agents shall be limited or even avoided. Future research to find safer alternatives for those food additives is recommended.
\end{abstract}

Keywords: beef, carcinogen, food additives, preservatives, processed beef

\begin{abstract}
ABSTRAK
Daging sapi adalah salah satu daging merah favorit yang mengandung berbagai nutrisi penting. Pada produk daging sapi olahan, bahan tambahan pangan (BTP) digunakan untuk berbagai keperluan termasuk pengawet, mempertahankan kelembapan dan tekstur, meningkatkan rasa dan stabilitas warna. Tujuan penelitian ini adalah untuk mengidentifikasi komposisi BTP dari produk daging sapi olahan komersial lokal dan halal berdasarkan peraturan di Indonesia. Sampel yang dinilai berjumlah 40 berbagai produk daging sapi olahan komersial, BTP yang diamati adalah penguat rasa, pengawet, penstabil, antioksidan, pengatur keasaman dan pewarna. BTP yang digunakan dalam produk sosis sapi adalah penstabil (30,00\%), pengawet $(25,56 \%)$, penguat rasa $(15,56 \%)$, pewarna $(14,44 \%)$, antioksidan $(13,33 \%)$ dan pengatur keasaman (1,11\%). Pada produk bakso sapi adalah penstabil (50,00\%), penguat rasa (20,00\%), pengawet (15,00\%), antioksidan, pengatur keasaman dan pewarna (masing-masing 5,00\%). Pada produk burger sapi adalah penstabil (29,73\%), pengawet (24,32\%), pewarna (21,62\%), penguat rasa $(13,51 \%)$, antioksidan dan pengatur keasaman (masing-masing 5,41\%). Pada produk kornet sapi adalah pengawet (32,00\%), penguat rasa dan antioksidan (masing-masing 24,00\%), dan penstabil (20,00\%). Pada produk daging sapi asap adalah pengawet $(50,00 \%)$, penstabil $(37,50 \%)$ dan antioksidan $(12,50 \%)$. Satu-satunya BTP yang digunakan dalam produk abon sapi adalah penguat rasa (100\%). Peraturan BTP ada baiknya ditinjau ulang oleh pemerintah. Meskipun saat ini masih diijinkan dalam regulasi di Indonesia, penggunaan BTP yang bersifat karsinogen sebaiknya dibatasi atau bahkan dihindari. Penelitian di masa depan untuk menemukan alternatif yang lebih aman untuk BTP ini sangat dianjurkan.
\end{abstract}

Kata kunci: bahan tambahan pangan, daging sapi, daging sapi olahan, karsinogen, pengawet

${ }^{*}$ Corresponding Author:

E-mail: mirza.zulkarnain@iuli.ac.id 


\section{INTRODUCTION}

Red meats contain various essential macronutrients and micronutrients. Beef is one of the favorite red meat. Lean raw beef is high-quality protein meat with various essential fatty acids, minerals (iron, zinc, selenium, sodium and potassium) and various B-vitamins including niacin, thiamin and B-12 (Wyness et al., 2011). The abundance source of protein, vitamins and minerals in beef makes it extensively and commercially used to prepare different beef dishes and processed beef products. Beef sausages, beef balls, beef burgers, corned beef, smoked beef and beef floss, each product has a unique flavor and texture which are desirable to consumers based on their preferences. Meat that has been changed through several processes such as salting, curing, smoking and fermentation for preservation or flavor improvement is called processed meat (Bouvard et al., 2015). Such products contain many different kinds of ingredients and food additives to give these varied meat products their distinctive taste and characteristics profile. Recently, researchers have realized the importance to distinguish between unprocessed and processed red meats (Binnie et al., 2014).

Food additives are any substances added to food to influence the characteristics or form of food (PMK, 2012). They are intentionally added to meat to prevent spoilage and oxidation, improve or maintain the flavor, texture, appearance and nutritional value of meat. Nowadays, the chemical compositions of food additives have become an important issue. There are growing concerns from the consumers regarding the composition and potential harmful factors for health in meat products (Mihaiu et al., 2015).

Despite some concerns about the safety issues on consumers' health, many food additives have been studied and proven safe as long as the regulations are followed. One of the internationally accepted tools to estimate the safe levels of food additives is the acceptable daily intake (ADI) which is the maximum amount of substance in milligrams per kilogram of body weight that can be consumed daily for a lifetime without causing any adverse effects on health. The lower the ADI indicated the higher the potential risk of toxicity of the substance. The maximum limit is the maximum number of food additives allowed to be found in the food in the units specified. Some food additives have the maximum limit good manufacturing practice (GMP), or cara produksi pangan yang baik (CPPB) meaning the amount of food additives is permitted in the sufficient quantities of food needed to produce the desired effect. Some of the food additives also have generally recognized as safe (GRAS) status. GRAS is a list of food additives that were considered safe for consumption, therefore they were allowed to use for commercially food products without special clearance. One important exception is Delaney Clause which is a clause to the 1958 Food Additives Amendment of the Pure Food and Drug Act in the United States that prohibits any food additives shown to cause cancer (carcinogenic) in laboratory animals or humans (Felter et al., 2020).

International Agency for Cancer Research (IARC) stated that if there is evidence of exposure to humans and some evidence or suspicion of carcinogenicity, the agents will be selected for review. According to the latest update on IARC Monograph (2019), there are 120 agents classified as group 1 (carcinogenic to humans), 82 agents classified as group 2A (probably carcinogenic to humans), 311 agents classified as group 2B (possibly carcinogenic to humans) and the other 500 agents are classified as group 3 (not classifiable as to its carcinogenicity to humans).

Recently, more than 800 epidemiological studies were reviewed by twenty-two experts from 10 countries (Bouvard et al., 2015). There were positive associations between consumption of red meat and processed meat with the incidence of cancer. The consumption of red meat is listed as a group 2A carcinogen while the consumption of processed meat is listed as a group 1 carcinogen (IARC, 2019). The risk of colorectal cancer will be $18 \%$ increased by eating 50 grams of processed meat daily and $17 \%$ increased by eating $100 \mathrm{~g}$ of red meat daily (Chan et al., 2011). Curing, smoking and other meat processing can lead to the formation of carcinogenic chemicals. Even though cooking improves the palatability and digestibility of meat, it also can produce $\mathrm{N}$-nitroso compounds (NOC), heterocyclic aromatic amines (HAA) (Alaejos and Afonso, 2011) and polycyclic aromatic hydrocarbons (PAH) (Alomirah et al., 2011) which are carcinogens.

Consumer's awareness of food additives in processed meat products is elevating due to the possible detrimental effects on health and wellbeing. Consumers commence to opt for a healthier lifestyle thus demand for less artificially preserved meats is also gradually increasing. Therefore, the consumers need to be educated about this risk and how some of the food additives used in processed meat can enhance the risk of carcinogens and other potential health problems. A study conducted by Fadlillah et al. (2015) in Bogor, Indonesia showed that there are still respondents who do not read the label and do not know the term food additives. Consumers with higher education and income tend to read labels more frequently while food additives information on the labels can influence purchasing decisions on most respondents who read labels. In Indonesia, the food additives usage is regulated by Ministry of Health (Peraturan Menteri Kesehatan 
Republik Indonesia Nomor 033 Tahun 2012) and Badan Pengawas Obat dan Makanan Republik Indonesia (BPOM RI). The purpose of this study is to identify the composition of food additives found in comercially processed beef products based on regulations in Indonesia. Awareness about the food additives composition in commercially processed beef products can influence purchasing decisions of these products in the future.

\section{MATERIALS AND METHODS}

\section{Materials}

The materials used for this study were all available commercially processed beef products obtained from various supermarkets in Jakarta and South Tangerang, Indonesia that have MD numbers registered to BPOM $\mathrm{RI}$ and have halal label approved by Majelis Ulama Indonesia (MUI). There were 40 commercially processed beef products obtained from 23 different brands of the following sorts: 18 sausages, 5 beef balls, 6 beef burgers, 6 corned beef, 2 smoked beef, and 3 beef floss products.

\section{Methods}

Each product was assessed for its food additive composition listed on the food labels. The percentage calculation of each type of food additive was based on the frequency of written composition of certain types of food additives in the food labels divided by the total food additives used in each product's category. The food additives assessed in this study were the food additives listed in the Regulation of The Ministry of Health of the Republic of Indonesia: Peraturan Menteri Kesehatan (PMK) Republik Indonesia Nomor 033 Tahun 2012. This regulation is also used as the reference to determine the function of each food additive used in the processed beef products. All the data were compiled and categorized based on its food category using Peraturan Kepala BPOM RI No. 21 Tahun 2016 tentang Kategori Pangan as the reference.

\section{RESULTS AND DISCUSSION}

\section{Beef sausages}

Beef sausages are products made from raw beef mashed with or without the addition of other food ingredients and put into sausage casings (BPOM, 2016). It is included in food category 08.3 (processed comminuted meat, poultry, and game products). There are 18 beef sausages products from 14 different brands that were assessed in this study.

The food additives that commonly used in beef sausages are flavor enhancers (including mono- sodium glutamate (MSG), disodium inosinate, disodium guanylate and disodium 5-ribonucleotide), preservatives (including sodium nitrite, sodium nitrate, sulfite, potassium sorbate, nisin and sodium benzoate), stabilizers (including various types of phosphate, dextrose, modified starch, carboxymethyl cellulose (CMC), carrageenan and non-specified vegetable stabilizers), antioxidants (including sodium erythorbate, tertiary butylhydroquinone (TBHQ), ascorbic acid and sodium ascorbate), acidity regulator (sodium lactate) and colorants (including ponceau 4R Cl 16255, erythrosine Cl 45430, caramel class I and caramel class IV). Only 3 variants (with the same brand) of beef sausages that do not contain any food additive (Table 1). Based on Figure 1, the types of food additives most frequently used in beef sausages products were stabilizers (30.00\%), followed by preservatives $(25.56 \%)$, flavor enhancers $(15.56 \%)$, colorants $(14.44 \%)$, antioxidants $(13.33 \%)$ and the least one was acidity regulator (1.11\%).

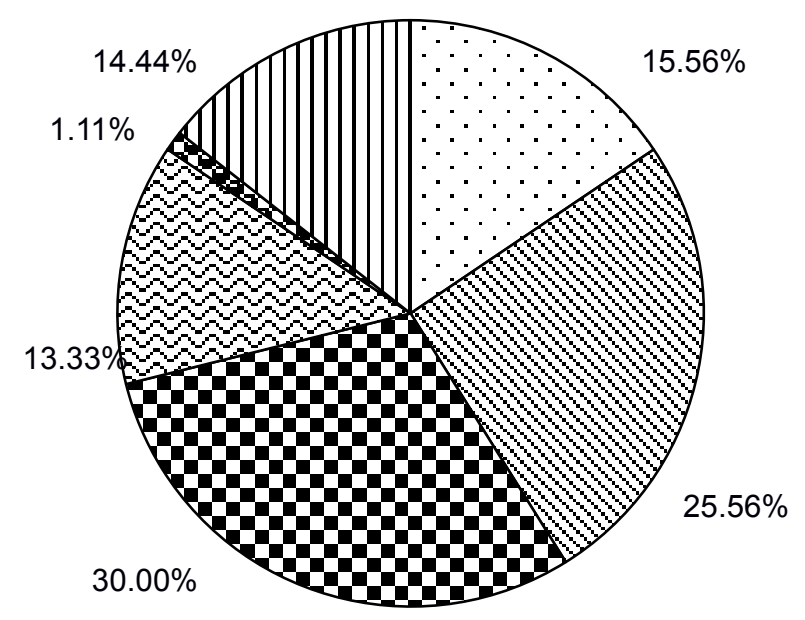

\begin{tabular}{|c|c|}
\hline $\begin{array}{l}\text { 口Flavor Enhancer } \\
\text { QStabilizer } \\
\text { ه Acidity Regulator }\end{array}$ & $\begin{array}{l}₫ \text { Preservative } \\
₫ \text { Antioxidant } \\
\square \text { Colorant }\end{array}$ \\
\hline
\end{tabular}

Figure 1. The types of food additives in beef sausages products

\section{Beef balls}

Meatball (bakso daging) is a processed meat product made from livestock meat mixed with starch and spices, with or without the addition of other food ingredients, which are round or other forms and cooked (BPOM, 2016). Beef balls are meatballs made from beef. They are also included in the food category 08.3. There are 5 beef balls products from 5 different brands that were assessed in this study. (Table 2). 
Table 1. Food additives usage in beef sausages products

\begin{tabular}{|c|c|c|c|c|c|c|c|}
\hline \multirow[b]{2}{*}{ No. } & \multirow[b]{2}{*}{ Brand } & \multicolumn{6}{|c|}{ Food Additive } \\
\hline & & $\begin{array}{c}\text { Flavor } \\
\text { Enhancer }\end{array}$ & Preservative & Stabilizer & Antioxidant & $\begin{array}{c}\text { Acidity } \\
\text { Regulator }\end{array}$ & Colorant \\
\hline 1 & PS1 & $\begin{array}{l}\text { Monosodium } \\
\text { glutamate } \\
\text { (MSG) }\end{array}$ & $\begin{array}{c}\text { Sulfite, Sodium } \\
\text { nitrite }\end{array}$ & $\begin{array}{c}\text { Disodium } \\
\text { diphosphate, } \\
\text { Sodium } \\
\text { tripolyphosphate, } \\
\text { Carrageenan }\end{array}$ & $\begin{array}{c}\text { Sodium } \\
\text { erythorbate, } \\
\text { Tertiary- } \\
\text { butylhydroquinone } \\
\text { (TBHQ) }\end{array}$ & & $\begin{array}{l}\text { Ponceau } \\
\text { 4R Cl } \\
16255 \\
\text { Erythrosine } \\
\text { Cl } 45430\end{array}$ \\
\hline 2 & PS2 & $\begin{array}{l}\text { Monosodium } \\
\text { glutamate } \\
\text { (MSG) }\end{array}$ & $\begin{array}{l}\text { Sulfite, Sodium } \\
\text { nitrite }\end{array}$ & $\begin{array}{l}\text { Disodium } \\
\text { diphosphate, } \\
\text { Sodium } \\
\text { tripolyphosphate, } \\
\text { Carrageenan }\end{array}$ & $\begin{array}{l}\text { Sodium } \\
\text { erythorbate, } \\
\text { Tertiary- } \\
\text { butylhydroquinone } \\
\text { (TBHQ) }\end{array}$ & & $\begin{array}{l}\text { Ponceau } \\
4 \mathrm{R} \mathrm{Cl} \\
16255 \\
\text { Erythrosine } \\
\text { Cl } 45430\end{array}$ \\
\hline 3 & CS1 & $\begin{array}{l}\text { Monosodium } \\
\text { glutamate } \\
\text { (MSG) }\end{array}$ & & $\begin{array}{c}\text { Carboxy } \\
\text { methylcellulose } \\
\text { (CMC), Modified } \\
\text { starch }\end{array}$ & Ascorbic acid & & $\begin{array}{l}\text { Erythrosine } \\
\mathrm{Cl} 45430\end{array}$ \\
\hline 4 & BS1 & $\begin{array}{l}\text { Monosodium } \\
\text { glutamate } \\
\text { (MSG) }\end{array}$ & $\begin{array}{l}\text { Sulfite, Sodium } \\
\text { nitrite }\end{array}$ & Dextrose & Ascorbic acid & & \\
\hline 5 & FS1 & $\begin{array}{l}\text { Monosodium } \\
\text { glutamate } \\
\text { (MSG) }\end{array}$ & $\begin{array}{c}\text { Sulfite, } \\
\text { Potassium } \\
\text { sorbate, } \\
\text { Sodium nitrite }\end{array}$ & $\begin{array}{l}\text { Sodium } \\
\text { tripolyphosphate }\end{array}$ & & & $\begin{array}{l}\text { Ponceau } \\
\text { 4R Cl } \\
16255, \\
\text { Erythrosine } \\
\text { Cl } 45430\end{array}$ \\
\hline 6 & CS2 & $\begin{array}{l}\text { Monosodium } \\
\text { glutamate } \\
\text { (MSG) }\end{array}$ & Sodium nitrite & Carrageenan & $\begin{array}{l}\text { Sodium ascorbate, } \\
\text { Sodium erythorbate }\end{array}$ & $\begin{array}{l}\text { Sodium } \\
\text { lactate }\end{array}$ & \\
\hline 7 & TS1 & & $\begin{array}{l}\text { Potassium } \\
\text { sorbate, Nisin }\end{array}$ & Phosphate & Sodium erythorbate & & \\
\hline 8 & ES1 & $\begin{array}{l}\text { Monosodium } \\
\text { glutamate } \\
\text { (MSG), } \\
\text { Disodium } \\
\text { inosinate, } \\
\text { Disodium } \\
\text { guanylate }\end{array}$ & $\begin{array}{l}\text { Sodium nitrite, } \\
\text { Sodium } \\
\text { benzoate }\end{array}$ & $\begin{array}{l}\text { Sodium } \\
\text { tripolyphosphate }\end{array}$ & & & $\begin{array}{l}\text { Caramel } \\
\text { class I }\end{array}$ \\
\hline 9 & KS1 & $\begin{array}{l}\text { Monosodium } \\
\text { glutamate } \\
\text { (MSG) }\end{array}$ & Sodium nitrite & $\begin{array}{c}\text { Phosphate, } \\
\text { Vegetable } \\
\text { stabilizer, Dextrose }\end{array}$ & & & $\begin{array}{l}\text { Caramel } \\
\text { class IV }\end{array}$ \\
\hline 10 & KS2 & & Sodium nitrite & $\begin{array}{c}\text { Phosphate, } \\
\text { Vegetable stabilizer, } \\
\text { Dextrose }\end{array}$ & & & \\
\hline $\begin{array}{l}11 \\
12\end{array}$ & $\begin{array}{l}\text { BS1 } \\
\text { BS2 }\end{array}$ & & & & & & \\
\hline 13 & BS3 & & & & & & \\
\hline 14 & HS1 & $\begin{array}{l}\text { Monosodium } \\
\text { glutamate } \\
\text { (MSG) }\end{array}$ & Sodium nitrite & $\begin{array}{l}\text { Phosphate, } \\
\text { Carrageenan }\end{array}$ & & $\begin{array}{l}\text { Sodium } \\
\text { Lactate }\end{array}$ & \\
\hline 15 & SS1 & & Sodium nitrite & Phosphate & Sodium erythorbate & & $\begin{array}{c}\text { Caramel } \\
\text { class IV, } \\
\text { Ponceau } \\
4 \mathrm{R} \mathrm{Cl} \\
\text { 16255, } \\
\text { Erythrosine } \\
\text { Cl } 45430\end{array}$ \\
\hline 16 & NS1 & & Sodium nitrite & & & & \\
\hline 17 & KS1 & $\begin{array}{l}\text { Monosodium } \\
\text { glutamate } \\
\text { (MSG), } \\
\text { Disodium 5- } \\
\text { ribonucleotide }\end{array}$ & $\begin{array}{l}\text { Sodium nitrite, } \\
\text { Potassium } \\
\text { sorbate }\end{array}$ & $\begin{array}{c}\text { Disodium } \\
\text { diphosphate, } \\
\text { Sodium } \\
\text { tripolyphosphate, }\end{array}$ & & & $\begin{array}{l}\text { Ponceau } \\
4 \mathrm{R} \mathrm{Cl} \\
16255\end{array}$ \\
\hline 18 & YS1 & $\begin{array}{l}\text { Monosodium } \\
\text { glutamate } \\
\text { (MSG) }\end{array}$ & $\begin{array}{l}\text { Sulfite, } \\
\text { Potassium } \\
\text { sorbate }\end{array}$ & $\begin{array}{l}\text { Phosphate, } \\
\text { Carrageenan }\end{array}$ & $\begin{array}{c}\text { Tertiary - } \\
\text { butylhydroquinone } \\
\text { (TBHQ), Ascorbic } \\
\text { acid }\end{array}$ & & \\
\hline
\end{tabular}


Table 2. Food additives usage in beef balls products

\begin{tabular}{|c|c|c|c|c|c|}
\hline \multirow{2}{*}{ No } & \multirow{2}{*}{ Brand } & \multicolumn{4}{|c|}{ Food Additives } \\
\hline & & Flavor Enhancer & Preservative & Stabilizer & Antioxidant \\
\hline 1 & BB1 & $\begin{array}{c}\text { Monosodium glutamate } \\
\text { (MSG) }\end{array}$ & & $\begin{array}{c}\text { Sodium diphosphate, } \\
\text { Potassium } \\
\text { polyphosphate }\end{array}$ & \\
\hline 2 & EB1 & & Sodium benzoate & & \\
\hline 3 & MB1 & $\begin{array}{l}\text { Monosodium glutamate } \\
\qquad(\mathrm{MSG})\end{array}$ & Potassium sorbate & $\begin{array}{c}\text { Sodium tripolyphosphate, } \\
\text { tetrasodium diphosphat, } \\
\text { mono diglyseride fatty } \\
\text { acid }\end{array}$ & \\
\hline 4 & FB1 & $\begin{array}{l}\text { Monosodium glutamate } \\
\text { (MSG) }\end{array}$ & Potassium sorbate & Sodium tripolyphosphate & $\begin{array}{l}\text { Sodium } \\
\text { erythorbate }\end{array}$ \\
\hline 5 & ZB1 & $\begin{array}{c}\text { Monosodium glutamate } \\
\text { (MSG) }\end{array}$ & & $\begin{array}{l}\text { Fosfat, sodium } \\
\text { bicarbonate }\end{array}$ & \\
\hline
\end{tabular}

Based on Figure 2, the types of food additives most frequently used in beef balls products are stabilizers $(50.00 \%)$, followed by flavor enhancers $(20.00 \%)$, preservatives $(15.00 \%)$, and the least are antioxidants, acidity regulators and colorants $(5.00 \%$ each).

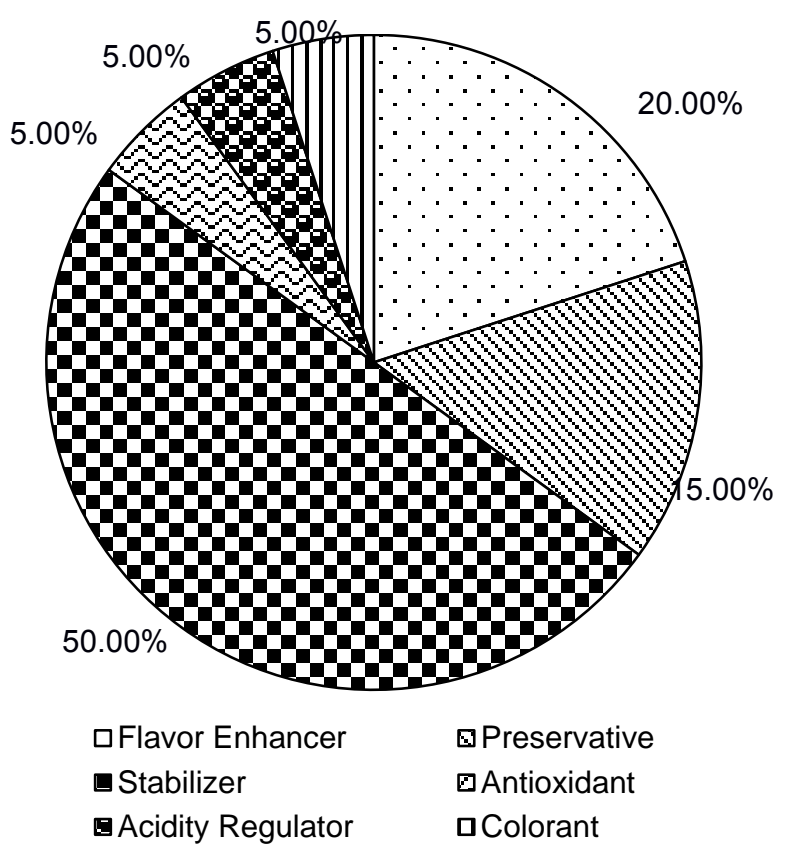

Figure 2. The type of food additives in beef balls products

\section{Beef burgers}

Beef burgers are products made from ground meat, with the addition of water, spices, with or without the addition of other food ingredients, then printed, rolled, cooked and cooled (BPOM, 2016). It is also included in food category 08.3. There are 6 beef burgers products from 5 different brands that were assessed in this study. Based on Table 3, the food additives that commonly used in beef burgers were flavor enhancers (monosodium glutamate (MSG)), preservatives (including sodium nitrite, sulfite and potassium sorbate), stabilizers (including various types of phosphate and modified starch), antioxidants (sodium erythorbate), acidity regulator (sodium lactate) and colorants (including Ponceau $4 \mathrm{R} \mathrm{Cl} 16255$ and Erythrosine $\mathrm{Cl}$ 45430). Based on Figure 3 , the types of food additives most frequently used in beef burgers products were stabilizers $(29.73 \%)$, followed by preservatives $(24.32 \%)$, colorants $(21.62 \%)$, flavor enhancers $(13.51 \%)$, and the least are antioxidants and acidity regulators (5.41\% each).

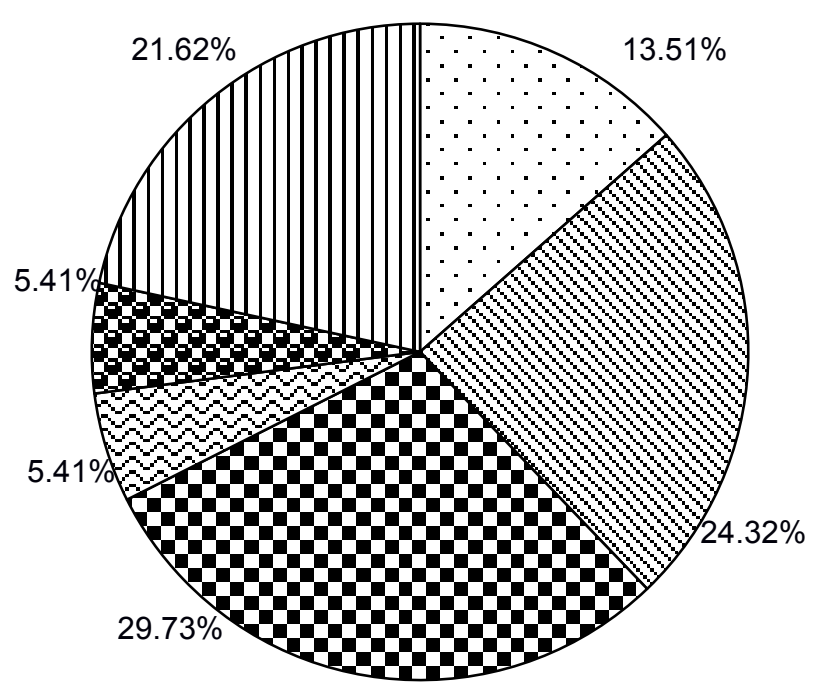

\section{Preservative \\ $\square$ Antioxidant \\ 口Colorant}

Figure 3. The types of food additives in beef burgers products 
Table 3. Food additives usage in beef burgers products

\begin{tabular}{|c|c|c|c|c|c|}
\hline \multirow{2}{*}{ No } & \multirow{2}{*}{ Brand } & \multicolumn{4}{|c|}{ Food Additives } \\
\hline & & Flavor Enhancer & Preservative & Stabilizer & Antioxidant \\
\hline 1 & BR1 & $\begin{array}{l}\text { Monosodium glutamate } \\
\text { (MSG) }\end{array}$ & Sodium nitrite & $\begin{array}{c}\text { Sodium diphosphate, } \\
\text { Potassium } \\
\text { polyphosphate }\end{array}$ & \\
\hline 2 & BR2 & & & $\begin{array}{c}\text { Sodium diphosphate, } \\
\text { Potassium } \\
\text { polyphosphate }\end{array}$ & \\
\hline 3 & FR1 & $\begin{array}{l}\text { Monosodium glutamate } \\
\text { (MSG) }\end{array}$ & $\begin{array}{c}\text { Suphite, Potassium } \\
\text { sorbate, Sodium } \\
\text { nitrite }\end{array}$ & Sodium tripolyphosphate & $\begin{array}{l}\text { Sodium } \\
\text { erythorbate }\end{array}$ \\
\hline 4 & KR1 & $\begin{array}{l}\text { Monosodium glutamate } \\
\text { (MSG) }\end{array}$ & $\begin{array}{l}\text { Potassium sorbate, } \\
\text { Sodium nitrite }\end{array}$ & Sodium tripolyphosphate & $\begin{array}{l}\text { Sodium } \\
\text { erythorbate }\end{array}$ \\
\hline 5 & AR1 & $\begin{array}{l}\text { Monosodium glutamate } \\
\text { (MSG) }\end{array}$ & Sodium nitrite & $\begin{array}{c}\text { Sodium diphosphate, } \\
\text { Potassium } \\
\text { polyphosphate, Modified } \\
\text { starch }\end{array}$ & \\
\hline 6 & VR 1 & $\begin{array}{l}\text { Monosodium glutamate } \\
\text { (MSG) }\end{array}$ & $\begin{array}{l}\text { Potassium sorbate, } \\
\text { Sodium nitrite }\end{array}$ & Sodium tripolyphosphate & \\
\hline
\end{tabular}

\section{Corned beef}

Corned beef is a product made from pieces of livestock or poultry meat and/or its mixture is boneless, fresh or frozen, can be mixed with the meat of the head and or heart, by curing, with or without additions (BPOM, 2016). It is also included in food category 08.3. There are 6 corned beef products from 4 different brands that were assessed in this study. Based on Table 4 , there are only 4 types of food additives that commonly used in corned beef, which are flavor enhancers (monosodium glutamate (MSG)), preservatives (including sodium nitrite, sulfite and potassium sorbate), stabilizers (including various types of phosphate and carrageenan) and antioxidants (sodium erythorbate). Based on Figure 4, there were only 4 types of food additives used in corned beef products, the most commonly used were preservatives (32.00\%), followed by flavor enhancers and antioxidants (24.00\% each), and the least one was stabilizers (20.00\%).

\section{Smoked beef}

Smoked beef is a product obtained by baking meat and/or curing meat or processed with permissible sources of smoke (wood bars, sawdust, or in the form of wood pellets and others, natural smoke) which are free of paint or wood preservatives (BPOM, 2016). It is included in Food Category 08.2 (Processed meat, poultry, and game products in whole pieces or cuts). There are 2 smoked beef products from 2 different brands that were assessed in this study. Based on Table 5, the food additives that are commonly used in the smoked beef products are preservatives (including sodium nitrite, sulfite and potassium sorbate), stabilizers (including various types of phosphate and non-specified vegetable stabilizers) and antioxidants (sodium erythorbate) (Figure 5). Based on Figure 5, there were only 3 types of food additives used in smoked beef products, the most commonly used were preservatives $(50.00 \%)$, followed by stabilizers $(37.50 \%)$ and the least one was antioxidants $(12.50 \%)$.

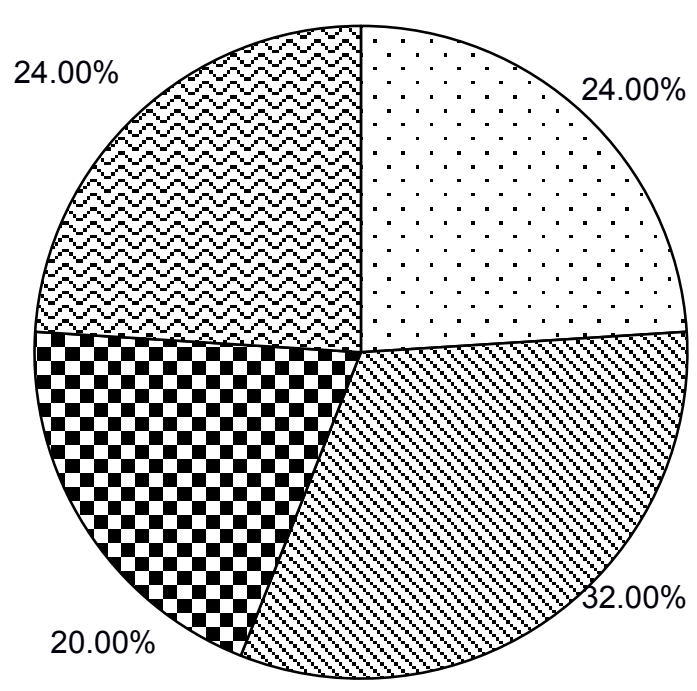

$$
\begin{array}{ll}
\square \text { Flavor Enhancer } & ₫ \text { Preservative } \\
\square \text { Stabilizer } & \square \text { Antioxidant }
\end{array}
$$

Figure 4. The types of food additives in corned beef products 
Table 4. Food additives usage in corned beef products

\begin{tabular}{|c|c|c|c|c|c|}
\hline \multirow{2}{*}{ No } & \multirow{2}{*}{ Brand } & \multicolumn{4}{|c|}{ Food Additives } \\
\hline & & Flavor Enhancer & Preservative & Stabilizer & Antioxidant \\
\hline 1 & PC1 & $\begin{array}{c}\text { Monosodium glutamate } \\
\text { (MSG) }\end{array}$ & Sodium nitrite & Sodium tripolyphosphate & $\begin{array}{c}\text { Sodium } \\
\text { erythorbate }\end{array}$ \\
\hline 2 & $\mathrm{PC} 2$ & $\begin{array}{c}\text { Monosodium glutamate } \\
\text { (MSG) }\end{array}$ & Sodium nitrite & Sodium tripolyphosphate & $\begin{array}{l}\text { Sodium } \\
\text { erythorbate }\end{array}$ \\
\hline 3 & PC3 & $\begin{array}{l}\text { Monosodium glutamate } \\
\text { (MSG) }\end{array}$ & Sulfite, Sodium nitrite & Sodium tripolyphosphate & $\begin{array}{l}\text { Sodium } \\
\text { erythorbate, } \\
\text { Ascorbic Acid }\end{array}$ \\
\hline 4 & $\mathrm{BC} 1$ & $\begin{array}{l}\text { Monosodium glutamate } \\
\text { (MSG) }\end{array}$ & Sodium nitrite & Carrageenan & \\
\hline 5 & $\mathrm{CC} 1$ & $\begin{array}{l}\text { Monosodium glutamate } \\
\text { (MSG) }\end{array}$ & & & \\
\hline 6 & $\mathrm{KC} 1$ & $\begin{array}{l}\text { Monosodium glutamate } \\
\text { (MSG) }\end{array}$ & $\begin{array}{l}\text { Potassium sorbate, } \\
\text { Sodium nitrite }\end{array}$ & Polyphosphate & $\begin{array}{l}\text { Sodium } \\
\text { erythorbate }\end{array}$ \\
\hline
\end{tabular}

Table 5. Food additives usage in smoked beef products

\begin{tabular}{|c|c|c|c|c|c|}
\hline \multirow{2}{*}{ No } & \multirow{2}{*}{ Brand } & \multicolumn{4}{|c|}{ Food Additives } \\
\hline & & Flavor Enhancer & Preservative & Stabilizer & Antioxidant \\
\hline 1 & BM1 & & Sodium nitrite & $\begin{array}{c}\text { Sodium diphosphate, } \\
\text { Potassium } \\
\text { polyphosphate }\end{array}$ & \\
\hline 2 & FM1 & & $\begin{array}{l}\text { Sulfite, Sodium } \\
\text { nitrite, Potassium }\end{array}$ & Vegetable stabilizer & $\begin{array}{c}\text { Sodium } \\
\text { erythorbate }\end{array}$ \\
\hline
\end{tabular}

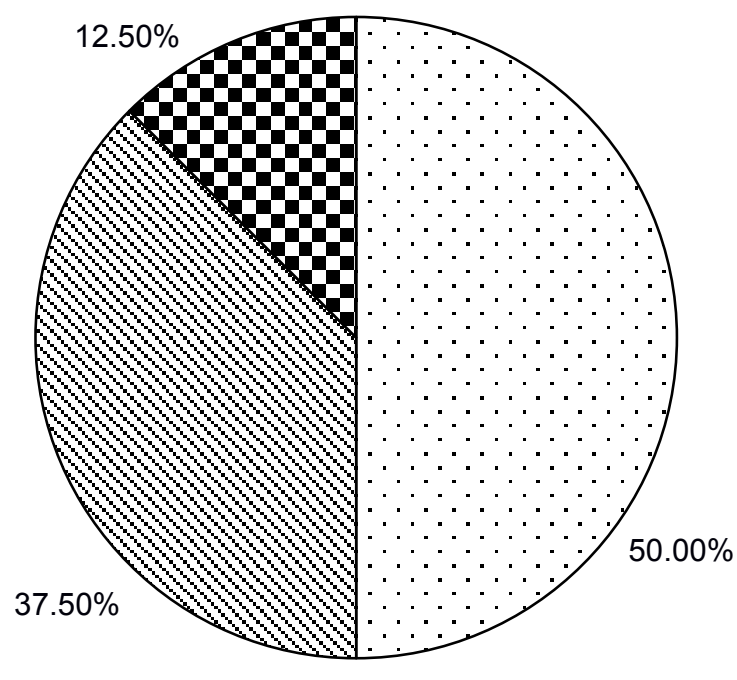

$\square$ Preservative $\quad$ Stabilizer $\square$ Antioxidant

Figure 5. The types of food additives in smoked beef products

\section{Beef floss}

Beef floss or Abon daging sapi is a typical dry food made from meat, boiled, sliced, seasoned, fried and can also be pressed, with a moisture content of not more than 7\% (BPOM, 2016). It is also included in Food Category 08.2. There are 3 beef floss from the same brands that were assessed in this study. Many beef floss products in the markets but only a few of them that have MD number, mostly are home industry (have P-IRT number) therefore they cannot be included in this study. Based on Table 6, the only food additives that are commonly used in beef floss products are flavor enhancer, which is monosodium glutamate (MSG).

Table 6. Food additives usage in beef floss products

\begin{tabular}{ccc}
\hline \multirow{2}{*}{ No } & \multirow{2}{*}{ Brand } & \multicolumn{1}{c}{ Food Additives } \\
\cline { 3 - 3 } & & Flavor Enhancer \\
\hline 1 & GF1 & Monosodium glutamate (MSG) \\
2 & GF2 & Monosodium glutamate (MSG) \\
3 & GF3 & Monosodium glutamate (MSG) \\
\hline
\end{tabular}

Based on Figure 6, three of the assessed beef floss only uses one category of food additive, which was flavor enhancer. Monosodium glutamate (MSG) was the ultimate flavor enhancer found in beef floss products. Based on the data collected in this study, the food additives that are commonly used for commercially processed beef products are flavor enhancers, preservatives, stabilizers, antioxidants, acidity regulators and colorants.

\section{Flavor enhancers}

Flavor enhancers are food additives to strengthen or modify the flavors and/or aromas that have been in food ingredients without giving new flavor and/or aroma (PMK, 2012). It enhances the existing taste and/or aroma of the food. Monosodium glutamate (MSG) is the most frequent flavor enhancer used for processed beef. Generally, MSG is an odorless, white crystalline powder that is added to food to give an umami taste. It is classified as GRAS by the Food and Drug Administration (FDA). 
ADI for MSG is not specified and its maximum usage is GMP.

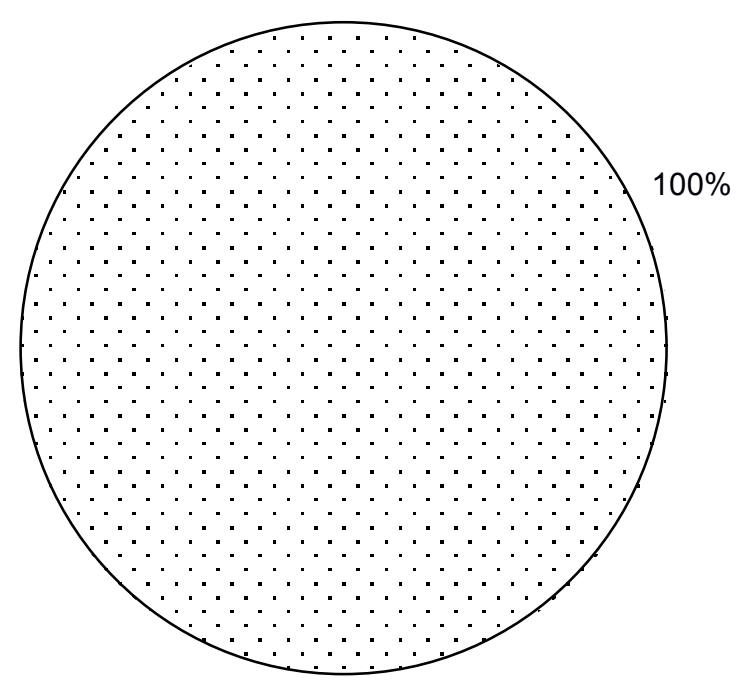

Flavor Enhancer

Figure 6. The types of food additives in beef floss products

However, some people are sensitive to the glutamate portion of MSG and can experience flushing, dizziness, sweating, chest pain, nausea, vomiting, headache, and other symptoms after exposure. Some of the natural ingredients intended to be added as the flavor enhancer but not listed in the list of food additives from Ministry of Health Regulation number 033 (PMK, 2012) such as hydrolyzed soy protein and yeast extract are excluded from the calculation of flavor enhancer in this study.

\section{Preservatives}

Preservatives are food additives to prevent or inhibit fermentation, decomposition, and other destruction of food caused by microorganisms (PMK, 2012). Basically, it prolongs the shelf-life of the food from deterioration caused by microorganisms. Nitrates and nitrites are the most frequent preservative used for processed beef, to fights harmful bacteria especially Clostridium botulinum and also lends them their pink coloration. However, studies show that ingested nitrate can be detrimental to health. Their consumption can lead to the synthesis of nitrosamines. Some nitrosamines are cancer-causing agents, especially for the stomach, colon and esophagus. The ingested nitrate or nitrite that results in endogenous nitrosation is classified as group 2A carcinogens (IARC, 2019). However, the United States Department of Agriculture (USDA) is the one who regulates the chemical usage in meats separate from those that govern Food and Drugs Administration (FDA) regulation in the United States (US). Delaney Clause does not go for USDA actions because they think no significant risk from the usage of nitrates and nitrites in meats. Decreasing the levels of nitrate and nitrite usage to the minimum required to achieve the preservative effect and also ensure microbiological safety in food products should minimize the nitrosamines exposure. The usage of potassium nitrite and sodium nitrite as food additives has been re-evaluated due to their potential carcinogenicity (EFSA, 2017). A review study conducted by Ferysiuk and Wójciak (2020) showed the possibility to use the combination of reduced nitrite and plant extracts to achieve similar product quality by using nitrite alone. For processed meats (food category 08.2 and 08.3), the maximum usage of nitrites (ADI 0-0.06 mg/kg body weight) is $30 \mathrm{mg} / \mathrm{kg}$ while the maximum usage of nitrates (ADI $0-3.7 \mathrm{mg} / \mathrm{kg}$ body weight) is 50 $\mathrm{mg} / \mathrm{kg}$. The very low ADI especially for nitrites indicated that we have to consider the usage limit of this additive very carefully to prevent any possible detrimental effect to health. Other preservatives used in commercially processed beef products assessed in this study were sulfite (ADI 0-0.7 mg/kg body weight), nisin (ADI 0-33000 unit/kg body weight), potassium sorbate (ADI $0-25 \mathrm{mg} / \mathrm{kg}$ body weight) and sodium benzoate (ADI 0-5 mg/kg body weight). Sulfites can cause hypersensitive reactions for a small subset of the population which can induce breathing difficulty, nausea and skin rashes (Robbins et al., 2015). Nisin is a bacteriocin that can be used as a natural preservative for many food products. It inhibits pathogenic bacteria such as Listeria monocytogenes and many other grampositive microorganisms in food (Gharsallaoui et al., 2016). Potassium sorbate and sodium benzoate are both listed as GRAS. The maximum limit of potassium sorbate usage is $1000 \mathrm{mg} / \mathrm{kg}$ (for processed meat food category 08.2 and 08.3 ).

\section{Stabilizers}

Stabilizers are food additives used to stabilize a homogenous dispersion system on food (PMK, 2012). It can help to maintain a uniform dispersion of two or more immiscible substances in food. The stabilizers used in the various processed beef products such as beef sausages, beef balls, beef burgers, corned beef and smoked beef are also possibly used as emulsifiers, thickeners, bulking agents and gelling agents in those products. According to Peraturan Menteri Kesehatan (PMK) Republik Indonesia Nomer 033 Tahun 2012, there are 104 types of approved stabilizers, 80 types of approved emulsifiers, 59 types of approved thickeners, 32 types of approved bulking agents and 10 types of approved gelling agents. The maximum 
usage of stabilizers such as mono and diglyceride of fatty acids, carbohydrate stabilizers such as carrageenan, carboxymethyl cellulose (CMC), modified starch, dextrose, and other non-specified vegetable stabilizers are mostly GMP. Therefore, there is no known health risk of such stabilizers when used properly. However, for phosphate stabilizers, such as disodium diphosphate, potassium polyphosphate, sodium tripolyphosphate, tetrasodium diphosphate, there is maximum tolerable daily intake (MTDI) of $70 \mathrm{mg} / \mathrm{kg}$ body weight, as phosphor $(P)$. The maximum limit of various phosphate for food category 8.3 is 2200 $\mathrm{mg} / \mathrm{kg}$ as total phosphor (P). Processed beef benefits from the use of phosphates in many ways include $\mathrm{pH}$ adjustment, buffering, sequestration of selected cations that can enhance the water holding capacity, texture and color, changing the ionic charges distributions and environment, bacteriostatic effects, inhibit lipid oxidation and shelf life extension (Long et al., 2011).

\section{Antioxidants}

Antioxidants are food additives to prevent or inhibit food damage due to oxidation (PMK, 2012). One of the primary causes of meat spoilage is oxidative degeneration therefore the oxidative stability is a key parameter in the estimation of meat quality (Candan and Bagdath, 2017). Falowo et al. (2014) added that lipid oxidation that leads to rancidity of fat, off-odors and flavors is the main cause of meat quality deterioration. Tertiary-butyl hydroquinone (TBHQ) is one of the various synthetic antioxidants commonly used in vegetable oil as one of the common ingredients in the processed beef. The ADI of TBHQ is $0-0.7 \mathrm{mg} / \mathrm{kg}$ body weight. Other frequently used antioxidants in processed beef products in this study are ascorbic acid and sodium erythorbate. ADI of both antioxidants is not specified. Ascorbic acid, also known as vitamin $C$ is included in GRAS and the maximum limit is GMP. While the maximum limit of sodium erythorbate is $500 \mathrm{mg} / \mathrm{kg}$ body weight. Sodium erythorbate decelerates the hydrolytic process and more effective in modified atmosphere packaging (MAP) products (KarpińskaTymoszczyk, 2013).

\section{Acidity regulators}

Acidity regulators are food additives for acidifying, neutralizing and/or maintaining food acidity (PMK, 2012). It can control the acidity or alkalinity of the food. From this research, sodium lactate is the most frequent acidity regulator used in commercially processed beef products. Besides acidity regulator, sodium lactate is also listed in the permitted humectant, thickener, bulking agent, emulsifier and stabilizer. It also has an antimicrobial effect but is not listed in the permitted preservatives in PMK no. 33 (2012). The result of a study done by Incoronato et al. (2016) indicated that the sodium lactate acted in synergy with modified atmosphere packaging (MAP) improving all the microbial quality. The ADI of sodium lactate is not limited and the maximum limit of sodium lactate is GMP, therefore it is not an additive to be concerned.

\section{Colorants}

Colorants are natural and synthetic dyes food additives, which when added or applied to food, can give or improve color (PMK, 2012). Both caramel class I and caramel class IV used for commercially processed beef products are naturally dark brown food colors, prepared by heating carbohydrates. The difference is caramel class IV is prepared with a presence of sulfite and ammonium ions, while caramel class I is prepared without them. Natural food colors are dyes made of plants, animals, minerals or other natural sources, including identical natural dyes through the process of extraction, isolation, or derivatization. ADI for caramel class I is not specified, while ADI for caramel class IV is $0-200$ $\mathrm{mg} / \mathrm{kg}$ body weight (in a liquid form) and 0-150 $\mathrm{mg} / \mathrm{kg}$ body weight (in a solid form). Both have the maximum limit GMP for processed meat products. On the other hand, Ponceau 4R Cl 16255 and Erythrosine Cl 45430 are synthetic red food colors. Synthetic food colors are dyes obtained by chemical synthesis. The ADI for Ponceau $4 \mathrm{R}$ and Erythrosine Cl No. 45430 is $0-4 \mathrm{mg} / \mathrm{kg}$ body weight and $0-0.1$ $\mathrm{mg} / \mathrm{kg}$ body weight, respectively. The maximum limit is $30 \mathrm{mg} / \mathrm{kg}$ for both Ponceau and Erythrosine (for food categories 08.2 and 08.3). Both Ponceau 4R and Erythrosine are included in 12 food dyes that were analyzed in meat products by lammarino et al. (2019) due to their safety concerns. Ponceau 4R is not permitted in the United States since some studies indicated the association between this synthetic color with attention deficit hyperactivity disorder (ADHD)-susceptible children. Erythrosine consumption is also related to altered cognition and behavior in children while different studies suggested the erythrosine consumption may increase the incidence of thyroid tumors. However, both of them are still included in the permitted food color in Indonesia (PMK, 2012). Studies done by MerinasAmo et al. (2019) concluded that a high chronic intake of food dyes throughout the entire life is not recommended. The exclusion of artificial food colorants from the diet may be beneficial to children with ADHD (Sonuga-Barke et al., 2013).

\section{CONCLUSION}

This study has identified the composition of food additives in commercially processed beef 
products. As presented in this study, the food additives that are commonly used for commercially processed beef products are flavor enhancers, preservatives, stabilizers, antioxidants, acidity regulators and colorants. Whether it is derived from the natural or synthetic compound, each food additive has its recommended purpose and they are beneficial to improve the quality attributes of processed beef products in terms of shelf life, flavor and appearance to consumers. The health concerns regarding the safety of food additives usage also need to be taken into account especially for food additives that are included in the list of carcinogens such as nitrites and nitrates that are still widely used in commercially processed beef products. Government Regulations on food additives may be reconsidered. Despite still allowed in Indonesian regulations at the present, food additives of which are included in the list of carcinogenic agents shall be limited or even avoided. Future research to find safer alternatives for those food additives is recommended.

\section{REFERENCES}

Alaejos MS, Afonso AM. 2011. Factors that affect the content of heterocyclic aromatic amines in foods. Compr Rev Food Sci F 10: 52-108. DOI: 10.1111/j.1541-4337.2010.00141.x.

Alomirah H, Al-Zenki S, Al-Hooti S, Zaghloul S, Sawaya W, Ahmed N, Kannan K. 2011. Concentrations and dietary exposure to polycyclic aromatic hydrocarbons (PAHs) from grilled and smoked foods. Food Control 22: 2028-2035. DOI: 10.1016/j.foodcont.2011.05.024.

Binnie MA, Barlow K, Johnson V, Harrison C. 2014. Red meats: time for a paradigm shift in dietary advice. Meat Sci 98: 445-451. DOI: 10.1016/j. meatsci.2014.06.024.

[BPOM] Badan Pengawas Obat dan Makanan. 2016. Peraturan Kepala BPOM RI No. 21 Tahun 2016 tentang Kategori Pangan. BPOM, Jakarta.

Bouvard V, Loomis D, Guyton KZ, Grosse Y, Ghissassi FE, Benbrahim-Tallaa L, Guha N, Mattock H, Straif K. 2015. Carcinogenicity of consumption of red and processed meat. Lancet Oncol 16: 1599-1600. DOI: 10.1016/ S1470-2045(15)00444-1.

Candan T, Bagdath, A. 2017. Use of natural antioxidants in poultry meat. CBU J Sci 13: 279 291.

Chan DSM, Lau R, Aune D, Vieira R, Greenwood DC, Kampman E, Norat T. 2011. Red and processed meat and colorectal cancer incidence:
Meta-analysis of prospective studies. PLoS One 6: 1-11. DOI: 10.1371/journal.pone.00204 56.

[EFSA] European Food Safety Authority. 2017. ReEvaluation of potassium nitrite (E 249) and sodium nitrite (E 250) as food additives. EFSA J 15: e04786. DOI: 10.2903/j.efsa.2017.4786.

Falowo AB, Fayemi PO, Muchenje V. 2014. Natural antioxidants against lipid-protein oxidative deterioration in meat and meat products - a review. Food Res Int 64: 171-181. DOI: 10.1016/j. foodres.2014.06.022.

Fadlillah HN, Nuraida L, Purnomo EH. 2015. Kepedulian konsumen terhadap label dan informasi bahan tambahan pangan (BTP) pada label kemasan pangan di kota Bogor. J Mutu Pangan 2: 119-126.

Felter SP, Llewelyn C, Navarro L, Zhang X. 2020. How the 62-year old delaney clause continues to thwart science: Case study of the flavor substance $\beta$-myrcene. Regul Toxicol Pharmacol 115: 104708. DOI: 10.1016/j.yrtph.2020.1047 08.

Ferysiuk K, Wójciak KM. 2020. Reduction of nitrite in meat products through the application of various plant-based ingredients. Antioxidants 9: 711. DOI: $10.3390 /$ antiox9080711.

Gharsallaoui A, Oulahal N, Joly C, Degraeve P. 2016. Nisin as a food preservative: Part 1: Physicochemical properties, antimicrobial activity, and main uses. Crit Rev Food Sci Nutr 56: 1262-1274. DOI: 10.1080/10408398.2013.7637 65.

lammarino M, Mentana A, Centonze D, Palermo C, Mangiacotti M, Chiaravalle AE. 2019. Simultaneous determination of twelve dyes in meat products: development and validation of an analytical method based on HPLC-UV-diode array detection. Food Chem 285: 1-9. DOI: 10.1016/j.foodchem.2019.01.133.

Incoronato AL, Gammariello D, Conte A, Del Nobile MA. 2016. Ready-to-cook fresh meal: Study for shelf life prolongation. J Food Sci Technol 53: 990-995. DOI: 10.1007/s13197-015-2136-1.

[IARC] International Agency for Research on Cancer WHO. 2019. IARC Monographs on the Identification of Carcinogenic Hazards to Humans: Preamble. Amended January 2019. Lyon, France.

Karpińska-Tymoszczyk M. 2013. The effect of oilsoluble rosemary extract, sodium erythorbate, their mixture, and packaging method on the quality of Turkey meatballs. J Food Sci Technol 50: 443-454. DOI: 10.1007/s13197-011-0359-3. 
Long NHBS, Gal R, Bunka F. 2011. Use of phosphates in meat products. Afr J Biotechnol 10: 19874-19882. DOI: 10.5897/AJBX11.023.

Merinas-Amo R, Martínez-Jurado M, Jurado-Güeto S, Alonso-Moraga Á, Merinas-Amo T. 2019. Biological effects of food coloring in in vivo and in vitro model systems. Foods 8: 176. DOI: 10.3390/foods8050176.

Mihaiu $M$, Tăbăran $A$, Mihaiu $R$, Dan $S$, Reget $O$, Cordiş I, Cordea D, Mureşan C. 2015. Compositional studies on some additive concentrations found in meat products marketed in a regional area. Bulletin UASVM Vet Med 72: 53-56. DOI: 10.15835/buasvmcn-vm:10567.

[PMK] Peraturan Menteri Kesehatan Republik Indonesia. 2012. Peraturan Menteri Kesehatan Republik Indonesia Nomor 033 Tahun 2012 tentang Bahan Tambahan Pangan.
Robbins KS, Shah R, MacMahon S, de Jager LS. 2015. Development of a liquid chromatographytandem mass spectrometry method for the determination of sulfite in food. J Agric Food Chem 63: 5126-5132. DOI: 10.1021/ff505525z.

Sonuga-Barke EJ, Brandeis D, Cortese S, Daley D, Ferrin M, Holtmann M, Stevenson J, Danckaerts $M$, van der Oord S, Döpfner M, Dittmann RW, Simonoff E, Zuddas A, Banaschewski T, Buitelaar J, Coghill D, Hollis C, Konofal E, Lecendreux M, Wong IC, Sergeant J. 2013. Nonpharmacological interventions for ADHD: Systematic review and meta-analyses of randomized controlled trials of dietary and psychological treatments. Amer J Psychiatry 170: 27589. DOI: 10.1176/appi.ajp.2012.12070991.

Wyness L, Weichselbaum E, O'Connor A, Williams EB, Benelam B, Riley H, Stanner S. 2011. Red meat in the diet: An update. Nutr Bulletin 36: 287. DOI: 10.1111/j.1467-3010.2010.01871.x. 